\title{
MODELING INTERRUPTIONS AND PATIENT FLOW IN A PREOPERATIVE HOSPITAL ENVIRONMENT
}

\author{
Bryan Pearce \\ Narges Hosseini \\ Kevin Taaffe \\ Clemson University \\ 110 Freeman Hall \\ Clemson, SC 29634, USA
}

\author{
Nathan Huynh \\ University of South Carolina \\ 300 Main St. \\ Columbia, SC 29208
}

\author{
Shannon Harris \\ Greenville Hospital System \\ 701 Grove Road \\ Greenville, SC 29605
}

\begin{abstract}
Late starting surgeries at a Greenville Memorial Hospital have been shown to cause process and scheduling disruptions, and are a major contributor to dissatisfaction among patients and hospital staff. The preoperative system requires the preparation of a high volume of patients, each with an individual set of characteristics and array of required tasks before surgery. Staff resources do not have a prescribed sequence of activities nor mutually exclusive duties. A novel discrete event modeling paradigm has been adopted for simulating the complex behavior of the preoperative system, identifying the underlying causes of process inefficiencies, and testing mitigating strategies. Current investigations are underway to shift the prescriptive approach of resource decision-making towards an agent-based approach, allowing resources to select their workload in such a way that achieves maximum utility for the agent.
\end{abstract}

\section{BACKGROUND AND LITERATURE}

Elective surgeries are the primary source of revenue generation at many hospitals, and it is widely observed that shrinking profit margins in healthcare is putting additional pressure on hospitals to increase surgical process throughput. At a local hospital, idle operating room (OR) time costs approximately $\$ 600 /$ hour, and overtime is prohibitively expensive. Opportunities for revenue generation without significant expenditures for capacity increases will require that the existing process for scheduling and executing be streamlined.

First case surgeries of the day are elective surgeries, both outpatient and inpatient, that have been scheduled to begin at 8:00 am. On-time starts has been a common performance indicator of hospital efficiency (Macario 2006). In fact, investigations have demonstrated that late starting first cases generate a ripple effect, by causing the following case in the same OR to be more frequently late or cancelled. This fact encouraged many researchers to find methods to calculate the cost per minute of tardiness in first cases of the day (e.g., McIntosh, Dexter, and Epstein 2006) or to study the impact of first on time starts on productivity (Overdyk et al. 1998). Since the tardiness of first starts increases the overall tardiness, and many hospitals have a high proportion of full-time staff rather than part time staff, the cost of 1 minute tardiness of a late first case is not always equal to the 1 minute of the labor costs (Macario and Dexter 2000). Each minute reduction in the tardiness of the first cases of the day in ORs with more than 8 hours of cases and turnover times results in $1.1 \pm 0.1$ minute reduction in regularly scheduled labor costs (Dexter and Epstein 2009). Cancelled and late cases prevent the maintenance of a stable schedule throughout perioperative services, and diminish potential opportunities to schedule more add-on cases. Buffers are 


\section{Pearce, Hosseini, Taaffe, Huynh and Harris}

built into the schedule in an attempt to predict and manage these disruptions, but these can overcompensate and create unexpected idle OR time.

Lapierre, Batson, and McCaskey (1999) built a measurement system that helps improve first on-time performance in health care organizations. They report that surgeons are the main reason that first cases are late, however the hospital's overall on-time performance provides motivation for improving surgeon performance and reliability. Wachtel and Dexter (2009) have also studied the factors that cause first case late starts. Although most of the previous research focused on the causes of the late first cases and the effects of these on efficiency, few practical solutions to the problem have been provided.

In this research, a discrete event simulation model was developed for simulating the complex behavior of the preoperative system, identifying the underlying causes of process inefficiencies, and testing mitigating strategies. Additional flexibility was incorporated by allowing resources to act as entities and have significantly more control over their actions. The next step in model development will be to allow resources to be in complete control of their actions using an agent-based modeling approach. Agent-based modeling is a quantitative methodology for modeling human behavior, and it has grown popular in recent years. It is a form of simulation modeling that allows individuals to respond based on the current environment, influencing the time required or method used to accomplish each activity. Sibbel and Urban (2001) discuss methods for abstraction of medical personnel behavior into algorithmic forms that can be implemented in an agent-based model, and issues surrounding the capture of the physical, emotional, cognitive, and social factors surrounding agents.

Agent-based modeling (ABM) also allows for the emergence of group/collective behavior as a result of the actions and interactions of the individual decision makers. This behavior is very evident in healthcare settings. Kanagarajah et al. (2006) have successfully implemented ABM by modeling the doctors, nurses, and other staff as self directing agents with behaviors dependent on demand loads, patient acuity, and other dynamic factors in an emergency department setting. Huang et al. (2010) consider the delivery of medical services from a higher perspective, and discuss communication, information horizons, and organizational memory concerns between distributed departments in a hospital.

Preop Holding is the site of all OR prep operations that do not take place in the OR itself, and the staff at Greenville Hospital System (GHS) identified the time required for patients to matriculate through the department as a process constraint. The research team has worked closely with GHS, through a series of data collection days and interviews, to gain an appropriate level of understanding to model such a system using simulation. As shown in Figure 1, outpatients require several tasks that inpatients do not. After arrival, outpatients first report to the receptionist for check in, and then sit in the waiting room until the business office, patient care technician (PCT), triage nurse, and phlebotomist are able to see them. Not all of these tasks are required for each outpatient, as some can be completed in pre-assessment. RNs will sometimes assist the triage nurse with her duties. After these tasks, the outpatient is escorted to their individual preop room by either the triage nurse or an RN. At this point, the processes for out/inpatients dovetail together. The patient is then visited by one or many of the PCT, Surgeon, Anesthesiologist, and Certified Registered Nurse Anesthetist (CRNA). Transport to the OR is accomplished by the CRNA once all of these tasks are complete, where the patient is prepped for surgery. There is opportunity to rearrange this process, but only to a limited degree. A map of task dependencies is shown in Figure 2 that illustrates which tasks must be completed before others.

\section{MODEL DEVELOPMENT}

\subsection{Data Sources}

Several sources of data were used to satisfy the parameter needs of the simulation model. Historical hospital databases were used to generate representative joint distributions for first case patient loads based on patient charge levels and inpatient/outpatient status. Upon investigation of the data, and through discussions with hospital staff, we can associate the patient charge level with an approximate patient acuity, so we adopted this terminology throughout the paper. Data from laboratory databases were extracted in order 


\section{Pearce, Hosseini, Taaffe, Huynh and Harris}

to accurately account for the return times of various blood work tests. The timestamps for Patient in Room (PIR) and Procedure Start Time (PST) were also harvested from past surgeries and used to create fitted distributions for patients of each acuity level.

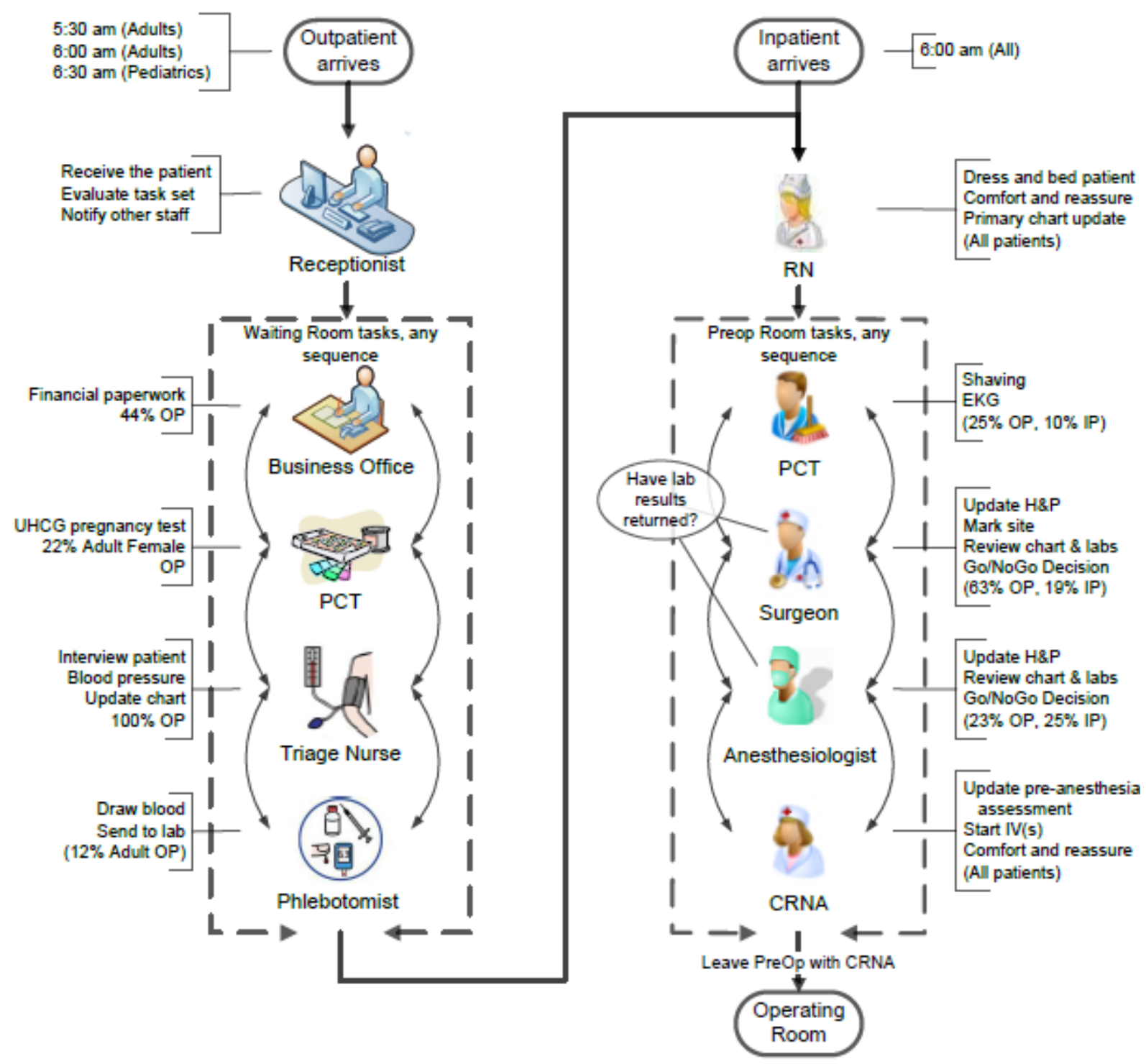

Figure 1: Process Flow Map.

No databases exist with time data from the processes in Preop Holding, so several days were devoted to shadowing patients through their stay and manually recording task times and idle intervals. These patients were tracked all the way until their procedures began, and the OR data collected were used to validate the OR timestamps from the database. These patient shadowing days were insufficient for revealing the behavior of the staff, however, so shadowing sessions were arranged with each resource. Structured interviews were conducted with each staff member shadowed.

Outpatient arrivals are scheduled at either 5:30,6:00, or 6:30 am. All adult outpatients are scheduled for either of earlier two time slots, and all pediatric outpatients are scheduled to arrive at 6:30. To allocate the 5:30 and 6:00 slots amongst the adult outpatient population, the scheduling method maintains an approximately even number of patients between the two slots while attempting to assign patients of acuity 
five or requiring blood tests to the 5:30 slot. All inpatients are scheduled to be brought to Preop Holding by support staff at 6:00.

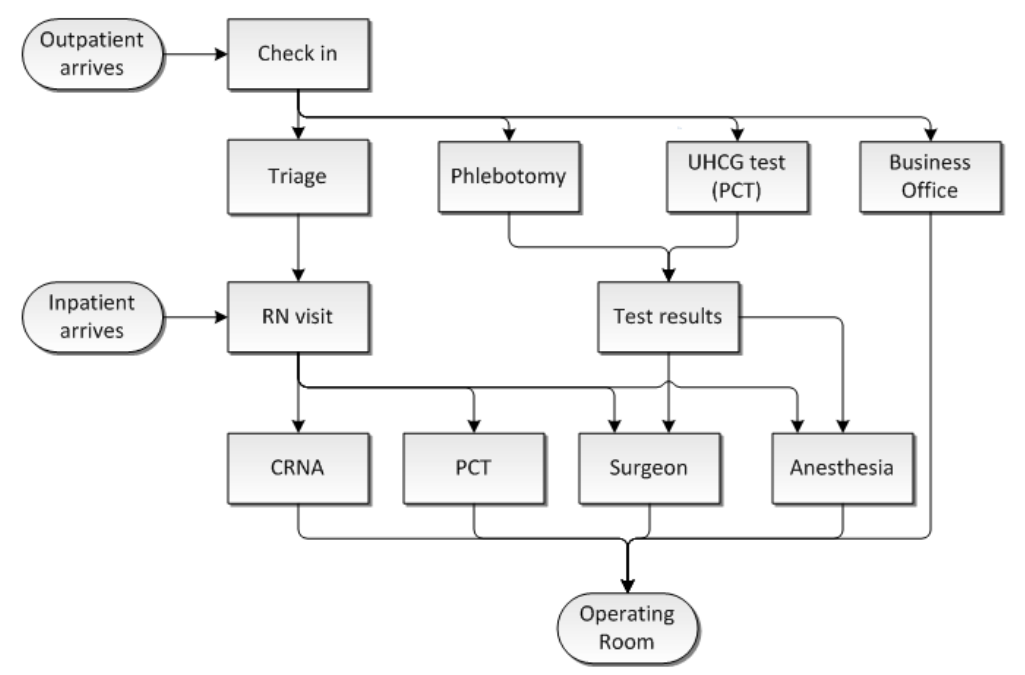

Figure 2: Task Dependency Map.

\subsection{Simulation Method}

All patients, staff, and informational units (charts, labs) in the system are modeled as independent entities, or agents within the model to simulate the flow and communication amongst them. Events on the atomical level are simulated using the Arena discrete event modeling engine. Staff task transition and communication logic is performed by considering the state of the system at task transitions. Figure 3 shows the decision making process for the RN upon completion of a task. Patients do not queue for service in a traditional DES manner, but instead progress through a series of pools. These pools are delineated by natural break points in the task dependency map, so that all patients in any pool are waiting for service by the same subset of staff resources. The pools used in the model are defined as:

- Waiting Room: Outpatients that have not yet been moved to their preop room.

- Preop Room 1: Patients that are in a preop room, but still require a subset of the outpatient specific tasks.

- Preop Room 2: Patients that await visits by their surgeon, anesthesiologist, CRNA, PCT, or lab return.

- OR: $\quad$ Patients that have been transported to the OR.

The selection of these pools permits staff resources to easily evaluate task selection by limiting the population of patients considered. When a resource determines which patient to work on next, the patient is pulled from the pool, batched with the resource for processing, and then returned to the appropriate pool when finished.

\section{CASE STUDY}

Analysis of the base model results identifies several delay metrics at various points in the process that contribute to the overall inefficiency of the system. The primary metrics of system performance used hereafter are defined as: 


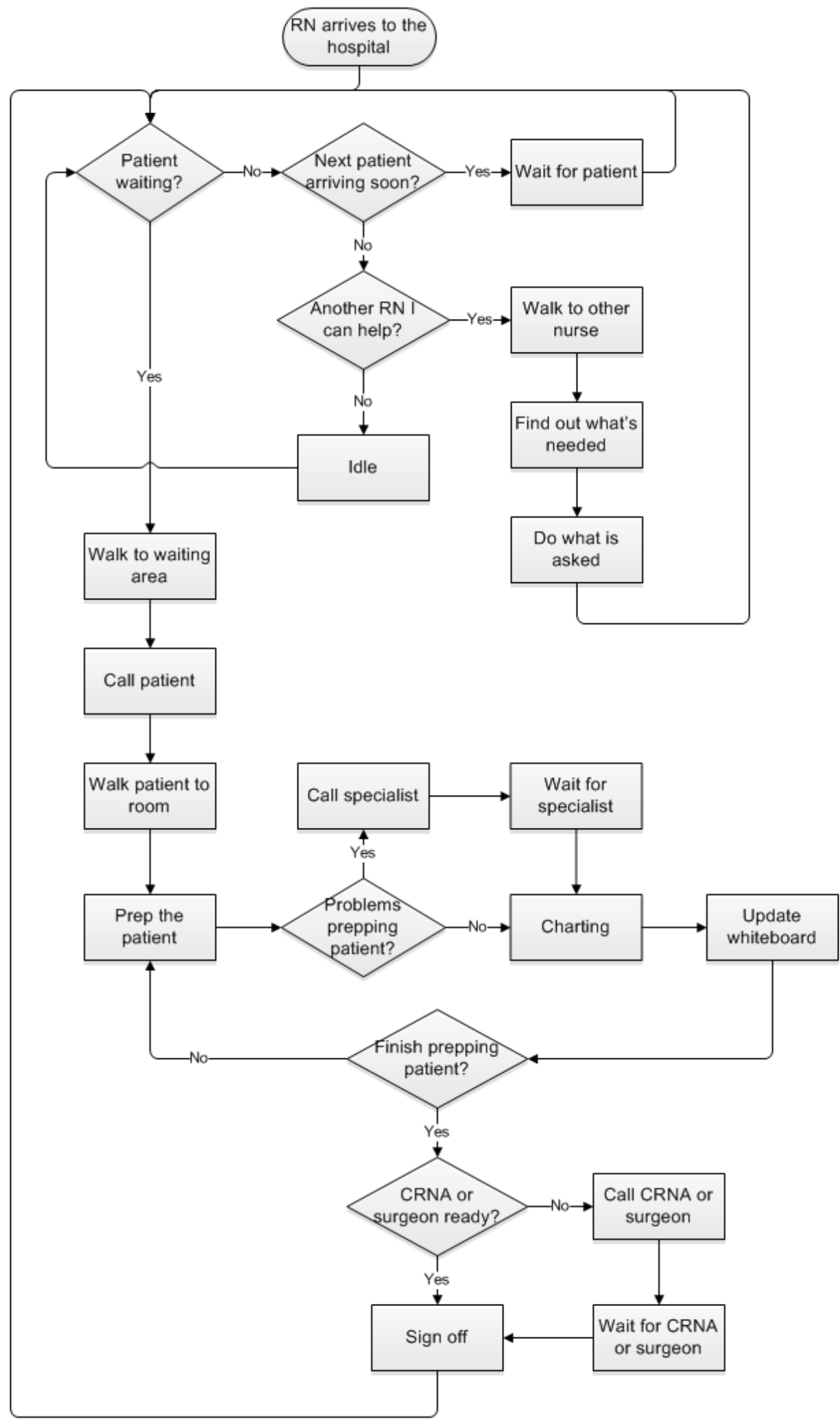

Figure 3. RN Decision Making Process. 


\section{Pearce, Hosseini, Taaffe, Huynh and Harris}

- Waiting Room dwell time is the duration of patient idle time in the waiting room.

- Bed Time is the time at which patients arrive to their preop room.

- Preop Ready is the time at which a patient has completed processing by all intra-preop tasks, requiring only visits from the surgeon, anesthesiologist, and/or CRNA before transport to the OR.

- Preop Ready Idle time is the duration of patient idle time after becoming Preop Ready.

- On Time ratio is the percentage of patient cases that start before 8:10 am.

These metrics were first investigated by performing sensitivity analysis on the model parameters that were thought to be relevant to delay at each stage of the process, in an effort to collect insights into the root causes of the problems.

In the waiting room average outpatient dwell time is 8.8 minutes, indicating the processes that pull patients from the waiting room contain a bottleneck. Independently varying patient needs for the phlebotomist, business office, triage, and urine human chorionic gonadotropin (UHCG) assay yielded a reduction in waiting room time only when reducing triage requirements. The data suggests that the triage process is likely the bottleneck, which supports the opinions gathered from RNs during interviews.

On average, 35 minutes of idle time is accrued per patient after becoming Preop Ready but before being transported to the OR. At this stage in the process the patient only lacks visits from the surgeon, anesthesiologist, and/or CRNA. The time at which these resources appear for patient visits is independent of other activities in preop holding, suggesting that this delay is directly attributable to other systems outside of the scope of preop holding. Sensitivity analysis on the time intervals that these final visits take place showed a strong correlation to both the Preop Ready Idle metric and On Time ratio.

The On Time ratio was also strongly correlated to the acuity level of the patient. Higher acuity levels require more time for OR preparation, as shown in Figure 4. OR preparation is the time spent in the OR prior to the start of the procedure (or the incision time).

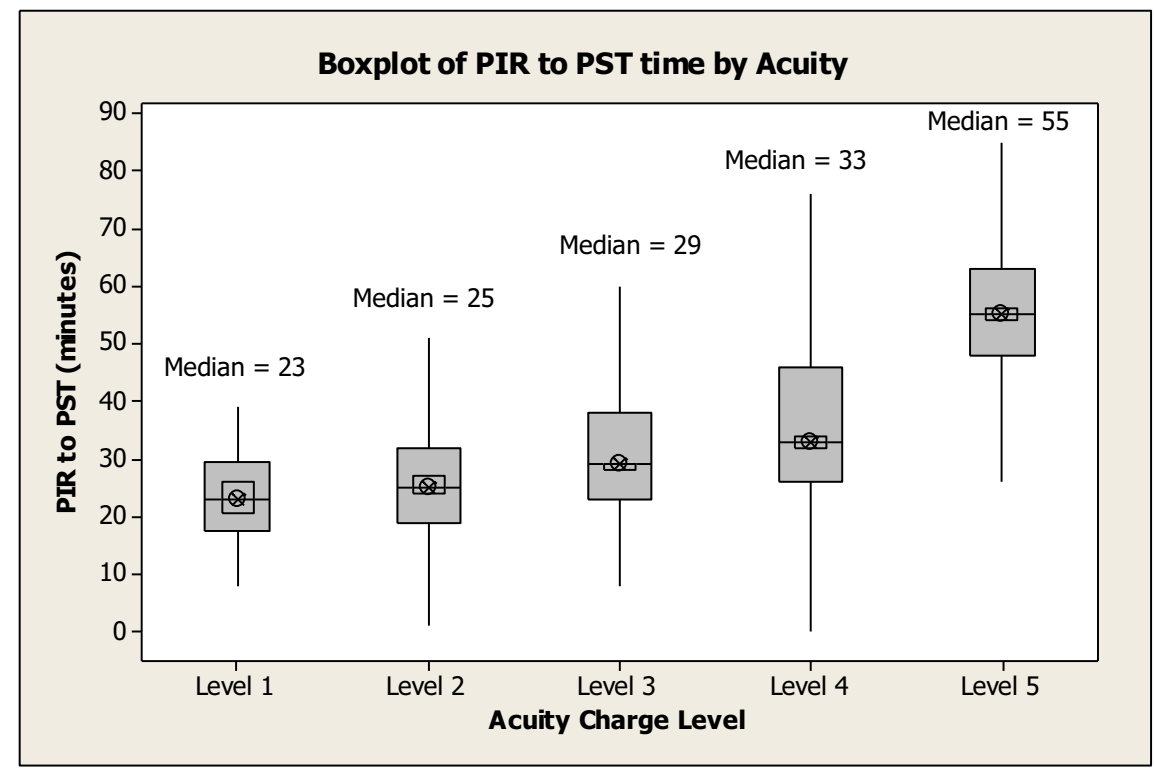

Figure 4. Breakdown of Pre-Surgery Time by Acuity Charge Level.

A set of several improvement scenarios were generated taking into account the insights gained from execution of the base model and by consideration of the responses from structured interviews with GHS staff. The task dependency map was used to discern task resequencing opportunities. Many of these scenarios were conceived to address specific phenomena observed in the base model. Screening experiments 


\section{Pearce, Hosseini, Taaffe, Huynh and Harris}

were conducted to identify the effects of the experimental factors, and then a subset of promising factors were used in the full factorial design.

Two factors were designed to control the scheduled arrival time of outpatient adults. One factor controls the number of these patients that are asked to come in at 5:30, and the other factor determines the scheduled arrival time of the rest of the outpatient adults. By permuting these scheduling factors any number of scheduling patterns can be achieved, as shown in Figure 5. The primary goal of these factors was to better sync outpatient arrivals with the system's capability to process them, in order to reduce waiting room time. The extreme policy of scheduling all arrivals at 5:30 was considered to be infeasible, due to waiting room concerns. Screening experiments did not show significant improvement in any performance metric when using these scheduling patterns. Presumably the large variance ( $\sigma=15$ min) of actual outpatient arrival time vs. scheduled arrival time acts to dampen the effects of these factors.

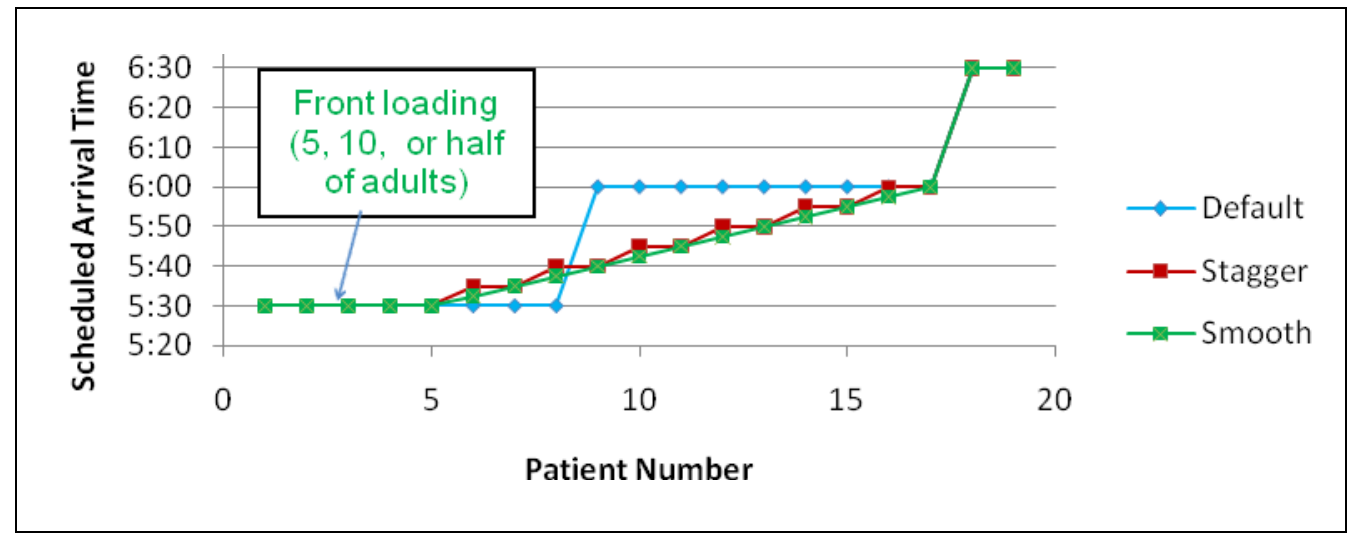

Figure 5. Patient Arrival Options to Test.

To control which outpatient adults would be assigned to these time slots, prioritization strategies were tested by considering permutations of weighting the patient's acuity level and bloodwork requirements. These control factors were designed to work with the longest processing time rule in mind, and attempt to schedule earlier arrival slots to patients that are known to require more processing time. Scenarios that placed priority on early arrivals for high acuity patients performed well in screening experiments, with bloodwork requirements showing a very mild effect. The most promising prioritization policy was to schedule acuity five patients first, following with acuity four, bloodwork patients, and then finally acuity three, two, and one patients.

There were three factors that determined alternative resource-to-task scenarios. In the first (FS1), one of the PCTs was reassigned from their usual duties to assist the triage nurse in processing waiting room patients. Another (FS2) absorbed the triage nurse into the RN staff, and mandated that all RNs would triage their own patients in the preop room. A third policy (FS3) was constructed that included all aspects of FS2, and additionally utilized the PCTs to move patients from the waiting room to the preop rooms.

To address the delay times after a patient becomes Preop Ready and the decoupled nature of the Surgeon, Anesthesiologist, and CRNA arrivals to Preop Holding activities, a scenario was created in which these resources would be sent a signal whenever their patient became Preop Ready. Instead of arriving according to their own preop-independent distributions, they arrive to Preop Holding to service their patient five minutes after the signal. The performance of these scenarios can be seen in Table 1 and Figure 6.

Using the RN staff to perform triage provides significant improvements in waiting room non-value added (NVA) time and preop ready time, as seen in the results of FS2 and FS3. The FS3 addition of tasking PCTs to escort patients from the waiting room to their preop rooms provides a small additional benefit to some performance metrics, and is non-dominated by the other task allocation scenarios. 
Table 1. Delays and On-Time ratios of resource tasking scenarios, with and without signaling.

\begin{tabular}{|l|c|cc|cc|}
\cline { 3 - 6 } \multicolumn{2}{c|}{} & \multicolumn{2}{c|}{ No Signal } & \multicolumn{2}{c|}{ Signal } \\
\hline Scenario & $\begin{array}{c}\text { Waiting Room } \\
\text { NVA time }\end{array}$ & $\begin{array}{c}\text { Preop Ready } \\
\text { NVA time }\end{array}$ & On Time \% & $\begin{array}{c}\text { Preop Ready } \\
\text { NVA time }\end{array}$ & On Time \% \\
\hline Base & 8.8 & 35.3 & $60.3 \%$ & 5 & $85.2 \%$ \\
FS1 & 22.7 & 26.2 & $49.3 \%$ & 5 & $67.3 \%$ \\
FS2 & 4.4 & 39.6 & $61.1 \%$ & 5 & $88.9 \%$ \\
FS3 & 0.4 & 41 & $59.8 \%$ & 5 & $89.9 \%$ \\
\hline
\end{tabular}

\section{Scenario Comparison}

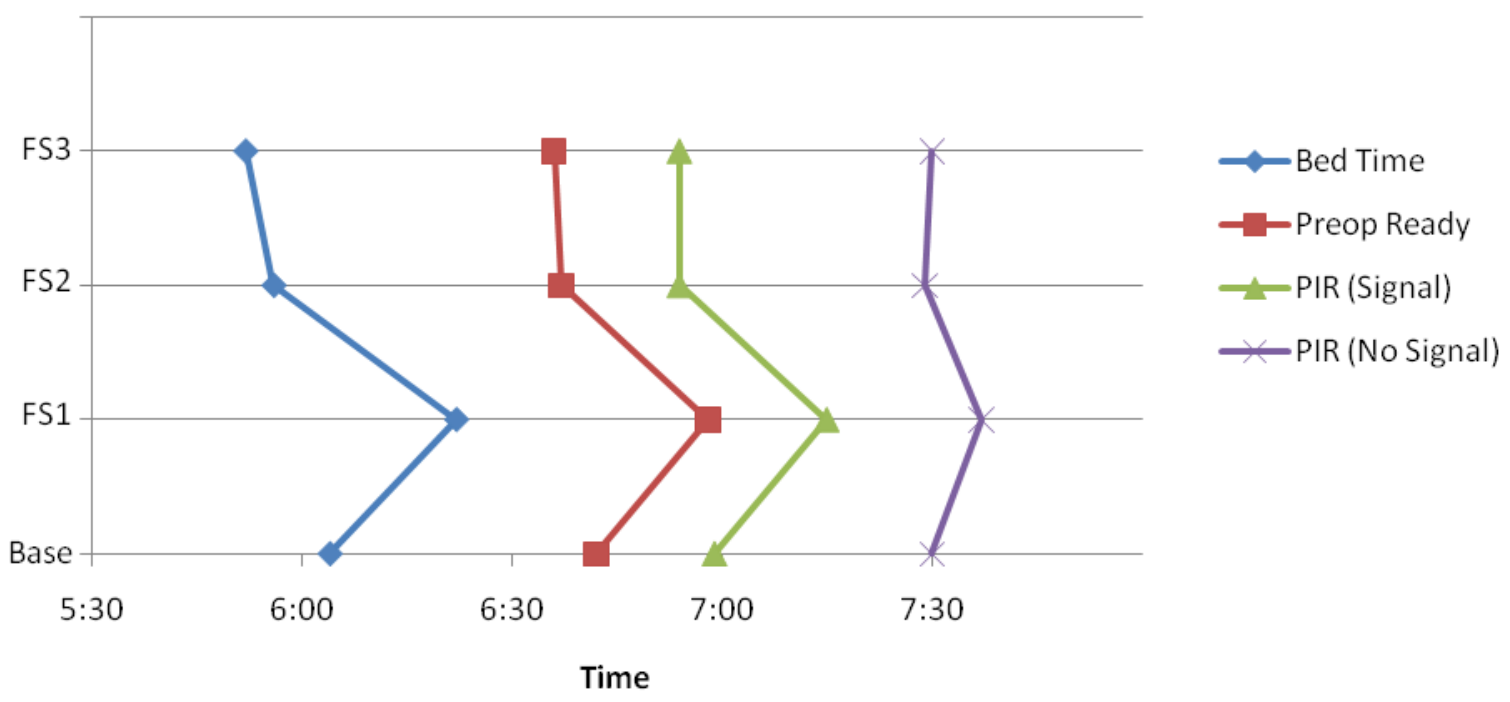

Figure 6. Threshold times of resource tasking scenarios, with and without signaling.

The signaling scenarios offer dramatic improvements to idle time intervals after patients are Preop Ready, and are the only scenarios found that can improve the percentage of cases that start on time. Specifically, both FS2 and FS3 (and, in fact, the base case) can all achieve an 85\% on-time start measure, which was considered to be the goal of GHS at the onset of the project.

As long as surgeons, anesthesiologists, and CRNAs arrive independently of their patient's progress through Preop Holding, the process is effectively bifurcated, as very few patients will incur additional delays after becoming Preop Ready. Signaling does not represent a process improvement solution insomuch as it is an indicator of the capability of the Preop Holding policies to deliver patients soon enough to be on time.

\section{ENHANCING THE MODEL USING AGENT-BASED RESOURCES}

The aforementioned model can be extended to better capture the complexity and dynamics involving the resources in the preop area. Of particular interest are how each resource chooses to go about performing his or her work and how their individual decisions affect the entire preop operations. To address this re- 


\section{Pearce, Hosseini, Taaffe, Huynh and Harris}

search problem, this study will seek to (1) model preop resources as utility-maximizing agents, (2) develop utility functions to capture the decision making criteria of each resource, and (3) investigate the effect of resource service strategy on patient flow. By utilizing an agent-based approach, the work performed by each resource can be modeled as follows.

1. Let $T$ be a set of tasks the resource can perform next (e.g. triage patient, assist other RN).

2. Select the task $t^{*}=\arg _{t \in T} \max u(t)$, where $u(t)=f\left(x_{1}, x_{2}, \cdots, x_{j}\right)$ and $x_{j}$ is the $\mathrm{j}^{\text {th }}$ criterion of interest (e.g. patient acuity, patient wait time).

It is noted that in this work, our focus is on developing utility functions to provide a descriptive model of current practice rather than a normative model. There currently exists an abundance of models that address clinical decision making, work allocation, communication, and management, with differing social and economic forces driving the choice of model. A comparison of the advantages and disadvantages of models and critiques of existing studies can be found in Tiedeman and Lookinland (2004).

Preop is a relatively small area and the layout is such that the resources work in close proximity with one another. Our observations of these resources indicate that there are often direct and indirect communications between them. Thus, in addition to modeling the individual resource's work tendency, this study will also consider interactions between the staff members. While it is beyond the scope of this paper to consider the social phenomenon such as "Organizational Communication" and "Collective Emotions," we will seek to draw conclusions on the effect of local interactions between the agents on the overall work flow.

\section{CONCLUSION}

In this paper, we were able to identify a key recommendation in how patient flow is handled within preop. Through the use of a signaling option that informs resources external to preop of their requested time to arrive to preop, a significant improvement in first case on-time starts can be achieved. This policy does not come without its difficulties, and it may require a shift in culture. However, the effect on process flow is clear. To this point, there are more modest gains in productivity through policy changes in how triage is performed for each patient.

Another outcome of this research is an investigation into an integrated agent-based model that can handle the complex nursing decisions that are made when caring for multiple patients. While the existing discrete event model has been developed to account for many of these decisions, the RNs do not have the ability to collectively act differently over time or respond as dynamically to their environment. For these reasons, an agent-based modeling framework is being developed.

\section{REFERENCES}

Dexter, F. and R. H. Epstein. 2009. Typical Savings from Each Minute Reduction in Tardy First Case of the Day Starts. Anesthesia \& Analgesia 108:1262-1267.

Jacintho, L., A. Batista, T. Ruas, M. Marietto, and F. Silva. 2010. An Agent-Based Model for the Spread of the Dengue Fever: A Swarm Platform Simulation Approach. In Agent Directed Simulation Proceedings, ed. L. Yilmaz. Orlando, FL.

Kanagarajah, A., P. Lindsay, A. Miller, and D. Parker. 2006. An Exploration into the Uses of AgentBased Modeling to Improve Quality of Health Care. In Proceedings of the 6th International Conference on Complex Systems, ed. A. Minai, D. Braha, Y. Bar-Yam. Boston, MA.

Lapierre, S., C. Batson, and S. McCaskey. 1999. Improving on-time performance in health care organizations: a case study. Health Care Management Science 2(1): 27-34.

Macario, A. and F. Dexter. 2000. Effect of Compensation and Patient Scheduling on OR Labor Costs. AORN Journal 71: 860-869. 
Macario, A. 2006. Are Your Hospital Operating Rooms "Efficient"?: A Scoring System with Eight Performance Indicators. Anesthesiology 105(2): 237-240.

McIntosh, C., F. Dexter, and R. Epstein. 2006. The Impact of Service-Specific Staffing, Case Scheduling, Turnovers, and First-Case Starts on Anesthesia Group and Operating Room Productivity: A Tutorial Using Data from an Australian Hospital. Anesthesia \& Analgesia 103:1499-1516.

Overdyk, F., S. Harvey, R. Fishman, and F. Shippey. 1998. Successful strategies for improving operating room efficiency at academic institutions. Anesthesia \& Analgesia 86:896-906.

Sibbel, R., and C. Urban. 2001. Agent-Based Modeling and Simulation for Hospital Management in Cooperative Agents. Applications in the Social Sciences. pp. 183-202. Available via:

<http://cuong.tgs.vn/ebook/Agent-

Based.Modeling.and.Simulation.for.Hospital. Management.pdf> [accessed April 15, 2010].

Tiedeman, M. E., and S. Lookinland. 2004. Traditional Models of Care Delivery: What Have We Learned? Journal of Nursing Administration 34(6): 291-297.

Tsai, C., S. Weng, C. Huang, C. Yang, J. Li, and K. Tsai. 2010. The Impact of Different Decision Behavior Models of Emergency Physicians on the Performance of Emergency Departments. In Emerging M\&S Applications in Industry and Academia Symposium Proceedings, Orlando, FL.

Wachtel, R., and F. Dexter. 2009. Influence of the Operating Room Schedule on Tardiness from Scheduled Start Times. Anesthesia \& Analgesia 108:1889-1901.

\section{AUTHOR BIOGRAPHIES}

BRYAN PEARCE is a PhD student in Industrial Engineering at Clemson University. His area of interests are in modeling complex systems, simulation, and healthcare. His email address is $<$ bpearce@clemson.edu>.

NARGES HOSSEINI is a PhD student in Industrial Engineering at Clemson University. Her area of interests are in combining simulation with optimization tools, specifically to analyze processes in the hospital environment. Her email address is <nhossei@clemson.edu>.

KEVIN TAAFFE is an Associate Professor of Industrial Engineering at Clemson University. Dr. Taaffe has been conducting research in healthcare logistics and emergency preparedness, transportation systems analysis, and inventory management. He supports the Clemson site for CELDi, the Center for Engineering Logistics and Distribution, where he works on industry-sponsored projects that bridge the gap between theoretical research and application. He is a member of IIE, INFORMS, SHS, and Transportation Research Board, and he serves on the Editorial Board for the International Journal of Operational Research. His email address is <taaffe@clemson. edu>.

NATHAN HUYNH is an Assistant Professor of Civil and Environmental Engineering at the University of South Carolina. Dr. Huynh's research focuses on improving processes in the areas of logistics and healthcare. He has a special interest in utilizing technology, modeling, and simulation to solve real-world problems. His email address is <huynhecec.sc.edu>.

SHANNON HARRIS is a Senior Management Engineer at the Greenville Hospital System (GHS). She has a Lean Six Sigma Black Belt for Service Certification. Her area of interests and expertise are in improving process and material flows, project management, labor management, and performance analytics. She is a member of IIE and SHS. Her email address is <sharriseghs. org >. 